\title{
GALAXY MOTIONS IN THE NEARBY UNIVERSE
}

\author{
JOHN P. HUCHRA \\ Harvard-Smithsonian Center for Astrophysics \\ 60 Garden Street, Cambridge, MA 02138 USA
}

\begin{abstract}
In this paper we review the history of the search for and study of the motions of nearby galaxies with respect to the Hubble Flow. The current status of the field is that (1) convincing infall has been detected into dense clusters, especially the Virgo cluster, (2) the microwave background direction is moderately well aligned with the measured flow nearby but not apparently on larger scales, and (3) there is good but not perfect consistency between the nearby density fields and velocity fields. Particular problems exist in the different $\Omega$ 's required to fit the density field derived from optically selected and IRAS $(60 \mu)$ selected galaxy samples.
\end{abstract}

\section{HISTORY}

The study of the uniformity of the expansion of the universe has a long an varied history which has only achieved a well defined focus in the last decade. Although there were several early attempts to search for distortions in the local flow field (e.g. Rubin 1951), the mythology that the local Hubble flow was basically smooth continued to hold sway in the astronomical community at-large throughout the early 1980's (e.g. Sandage and Hardy 1972). despite the work of Rubin and collaborators (Rubin et al. 1976a, 1976b).

This view changed dramatically in the late 1970's. There was a major paradigm shift brought on by two related events. First, the Cosmic Microwave Background (CMB) dipole was convincingly detected by a number of groups (e.g. Cheng et al. 1979; Smoot and Lubin 1979). This detection was so convincingly a dipole that, despite attempts to explain it via emission from galaxies or other cosmological effects, there was little doubt that a motion of the Solar sytem of $\sim 300 \mathrm{~km} / \mathrm{sec}$ with respect to the CMB frame had been detected and that a transformation of that motion into the 


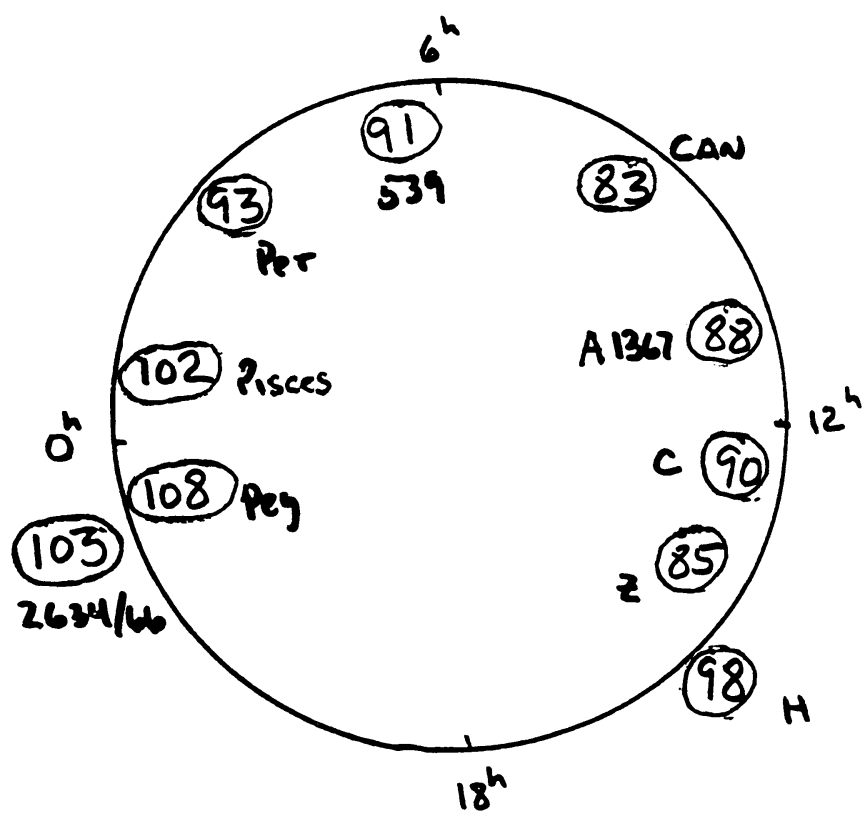

Figure 1. The Hubble constant towards medium distant clusters measured in the Arecibo declination zone. The "dipole" is indicative of a motion of $\sim 480 \mathrm{~km} / \mathrm{s}$ towards Virgo consistent with the CMB vector in this band. (based on Aaronson et al. 1980).

reference frame of the Local Group gave a motion w.r.t. the CMB frame of $\sim 600 \mathrm{~km} / \mathrm{s}$.

Second, evidence from high quality measurements of the relative distances of galaxies (made primarily to measure the Hubble Constant) began to indicate that motions in the nearby universe might exist. Aaronson et al. (1980) found that Infrared Tully-Fisher distance measurements to medium distant $(4-10,000 \mathrm{~km} / \mathrm{s})$ clusters of galaxies gave a Hubble constant nearer $95 \mathrm{~km} / \mathrm{s} / \mathrm{Mpc}$ than the $\sim 65 \mathrm{~km} / \mathrm{s} / \mathrm{Mpc}$ obtained for the nearby Virgo and Ursa Major clusters (Figure 1). This could be explained by an infall of the Local Group into Virgo of a few hundred $\mathrm{km} / \mathrm{sec}$ relative to a uniform Hubble flow. Furthermore, there was a dipole in the $\mathrm{H}_{0}$ measurements to the distant clusters that could also be explained by such a motion. This effect was also seen in measurements of relative distances to elliptical galaxies made by Tonry and Davis (1980) as well as other investigators.

The infall into Virgo, which had been predicted by numerous authors since the mid-1950's (de Vaucouleurs 1958; Peebles 1976; Silk 1974) was finally confirmed by Aaronson et al. (1982) with a detailed study of the flow field w.r.t. the Virgo Cluster interior to $3,000 \mathrm{~km} / \mathrm{s}$ (Figure 2). Aaronson 


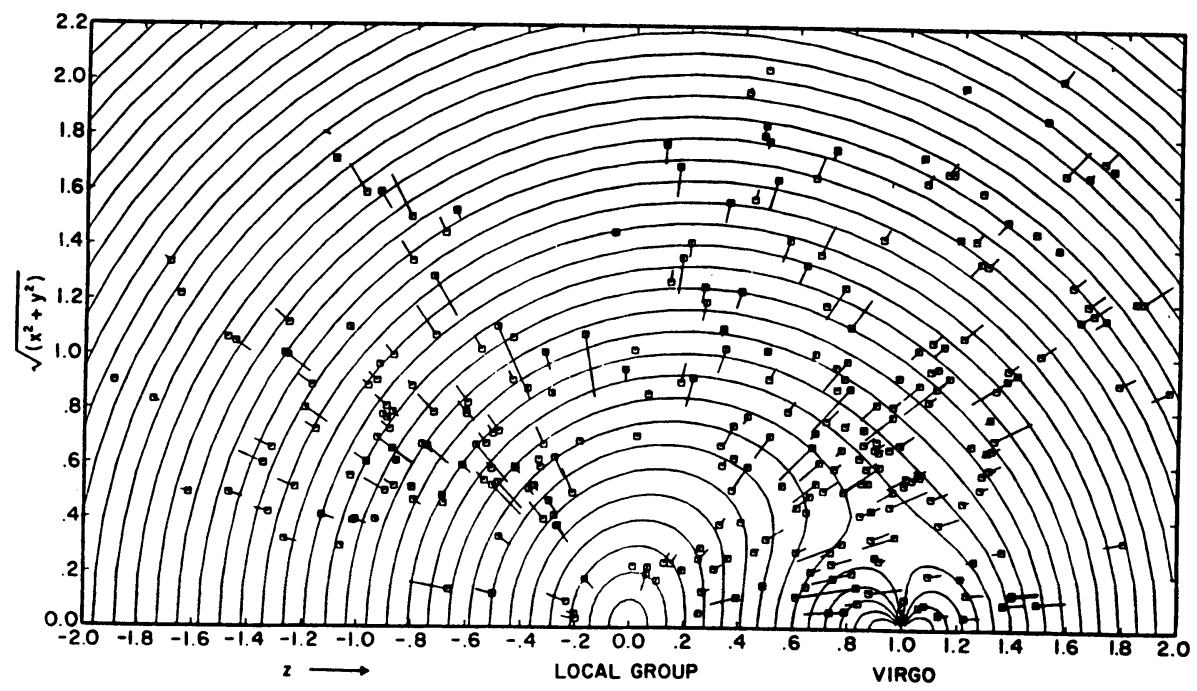

Figure 2. The infall pattern around the Virgo cluster matched to velocity-distance residuals from a uniform Hubble expansion (from Aaronson et al. 1982).

et al. detected the LG motion w.r.t. Virgo at the $5+\sigma$ level and were able to split it into two components, a "pattern" infall of $\sim 250 \mathrm{~km} / \mathrm{s}$ and a random component of $\sim 80 \mathrm{~km} / \mathrm{s}$.

All of these results were echoed by the detection of significant anisotropy in the galaxy distribution by the redshift surveys of the time (Sandage and Tammann 1981; Davis et al. 1982; Kirshner et al. 1981; c.f. Geller and Huchra 1989 for a more detailed review). Combined with the realization that the Universe not empty, i.e. that $\Omega \neq O$ and more likely between 0.2 and 1.0, this was strong proof that flows like the infall to Virgo must exist in the nearby Universe.

However, there was still a problem. Simply stated:

$$
\begin{aligned}
\overrightarrow{V i r g o} & \neq \overrightarrow{C M B} \text { by } \sim 45^{\circ}, \\
\text { and } V_{\text {Virgo }} & \neq V_{C M B} \text { by } 350 \mathrm{~km} / \mathrm{s} .
\end{aligned}
$$

The Virgo infall velocity and Virgo direction cannot be completely responsible for the CMB motion. This problem caused a number of people (Shaya 1984; Tully and Shaya 1984; Lilje, Yahil and Jones 1986; Tammann and Sandage 1985) to suggest that there was an additional density concentration causing the remaining component of the motion roughly in 
the direction of the Hydra-Centuarus Supercluster. Most of these authors suggested that Hydra-Cen might even be the gravitational cause of the additional component of our motion.

The solution to this problem seemed to be in sight when, in 1986, the "Seven Sammurai" (Burstein et al. 1986; Lynden-Bell et al. 1988) claimed a detection of a "Great Attractor" - an gravitating mass causing a bulk flow roughly towards Hydra-Centaurus and the CMB direction, from $\mathrm{D}_{n^{-}}$ $\sigma$ measurements of $\sim 400$ elliptical galaxies. However, the details of the motion were way off expectations - Hydra and Centaurus were themselves moving towards a point not coincident with their center-of-mass and large deviations, in excess of $1000 \mathrm{~km} / \mathrm{s}$, were seen in the flow field. Aarosnon et al. (1986) continued their work with the IRTF relation to map the flow field using clusters of galaxies rather than individual objects (thus beating down the relative distance errors by $\sqrt{n}$ ) and also found evidence for both an infall to Virgo and motion towards Hydra-Cen.

These works have set the stage for a large number of studies of local motions aimed at answering a range of fundamental questions related to flows, large-scale structure, $\Omega$ and cosmology in general.

\section{FUNDAMENTAL QUESTIONS}

The fundamental questions we are asking are relatively simple to state:

1. What is the local Flow Field? (a) Does it converge, and if so on what scale? (b) Can we explain the CMB dipole locally? (c) How does it effect the determination of the Hubble Constant, $\mathrm{H}_{0}$ ?

2. What is the local Density Field? (a). Can it explain the flow field? (b) If so, what $\Omega$ is required to do so?

Despite their simplicity, these are not easy questions to answer. The answer to the first set depends on the existence of accurate relative distance indicators. The best available are the Surface Brightness Fluctuation technique pioneered by Tonry (Tonry and Schneider 1988). This technique is gradually taking the place of the $\mathrm{D}_{n}-\sigma$ technique for nearby early type galaxies, but is time consuming (telescope time!) and hard to use at large distances (although HST can be used, again, if sufficient time can be made available). Other reasonably accurate methods include the IR and red (usually $\mathrm{R}$ band, $6500 \AA$, or I band, $8500 \AA$ ) Tully-Fisher method, useful for measuring distances of edge-on spirals, the $\mathrm{D}_{n}-\sigma$ technique and Supernovae (both type Ia's and type II's via the standard-candle approach now modified by light curve fitting (Hamuy et al. 1995; Reiss et al. 1995) or the Expanding Photospheres Method (Schmidt et al. 1994). Application of any or all of these techniques to all-sky flow field maps with relatively dense coverage is a long way away. 
Since the mid-1980's there have been a number of studies of the flow field. Most, unfortunately, have concentrated on small, well defined regions of the sky. Mathewson, Ford and Buchhorn (1992) and Mathewson and Ford (1994), have concentrated their studies in the region of the original Great Attractor and find a complicated and non-uniform flow over the region, probably not converging out to $8,000 \mathrm{~km} / \mathrm{s}$. Dressler and Faber (1991) dispute the early claims of Mathewson $e t$ al. and claim to find backside infall into the GA. Willick (1991) investigated the Perseus-Pisces region and found an infall of the Pisces-Perseus region towards us! Courteau (1992) rexamined this region and found evidence for both a bulk flow and a relatively quiet region in the Hubble flow above PP. Courteau et al. 1993 systhesize all the available peculiar velocity results to show that there may be a "large-scale parallel streaming" or bulk flow of all galaxies inside a $6000 \mathrm{~km} / \mathrm{s}$ sphere as well as the traditional GA. This conclusion has been strongly supported by the ground breaking work of Lauer and Postman (1994), who in a manner analogous to Sandage's work on the Hubble Diagram, used first ranked cluster galaxies to probe the velocity field out to $15,000 \mathrm{~km} / \mathrm{s}$. They too find evidence for a bulk flow on very large scales but one that is not consistent with the microwave background vector! Lauer and Postman find that the Abell Cluster Inertial Frame that they investigate appears to be moving with a velocity of $689 \pm 178 \mathrm{~km} / \mathrm{s}$ w.r.t. the CMB frame and that the motions (at least of clusters!) inside that ragion is relatively quiescent. This is also seen in the work of Mould et al. (1991; 1993) who find motion towards the GA (Figure 3), but a relatively quiet field inside $8,000 \mathrm{~km} / \mathrm{s}$ except for a "hiccup" at the position of the GA.

The answer to the second question requires unbiased (in the classical sense) samples of galaxies. Attempts have been made (e.g. POTENT Bertschinger and Dekel 1989; Dekel, Bertschinger and Faber 1990) to derive the density field from the velocity field. However, what one really wants to do is to determine the density field and velocity field independently so as to be able to perform the comparison between the two. In that case, one needs to construct an all-sky map of galaxies and measure approximate distances, usually via redshifts, to the galaxies. Unfortunately, the Milky Way gets in the way!

Most work on the density field since the early 1980's has been, almost by definition, on three samples of objects - (1) optically selected galaxies from the Zwicky, UGC and ESO catalogs, (2) infrared $(60 \mu)$ selected galaxies from the IRAS all sky survey, the point source catalog, and (3) galaxy clusters from the Abell catalog. The optical work, both on galaxies and clusters, is hampered by the effects of absorption by the dust in the Galaxy, but can produce exquisitely dense surveys (Figure 5), while the IRAS samples are much less well sampled and are biased against high den- 


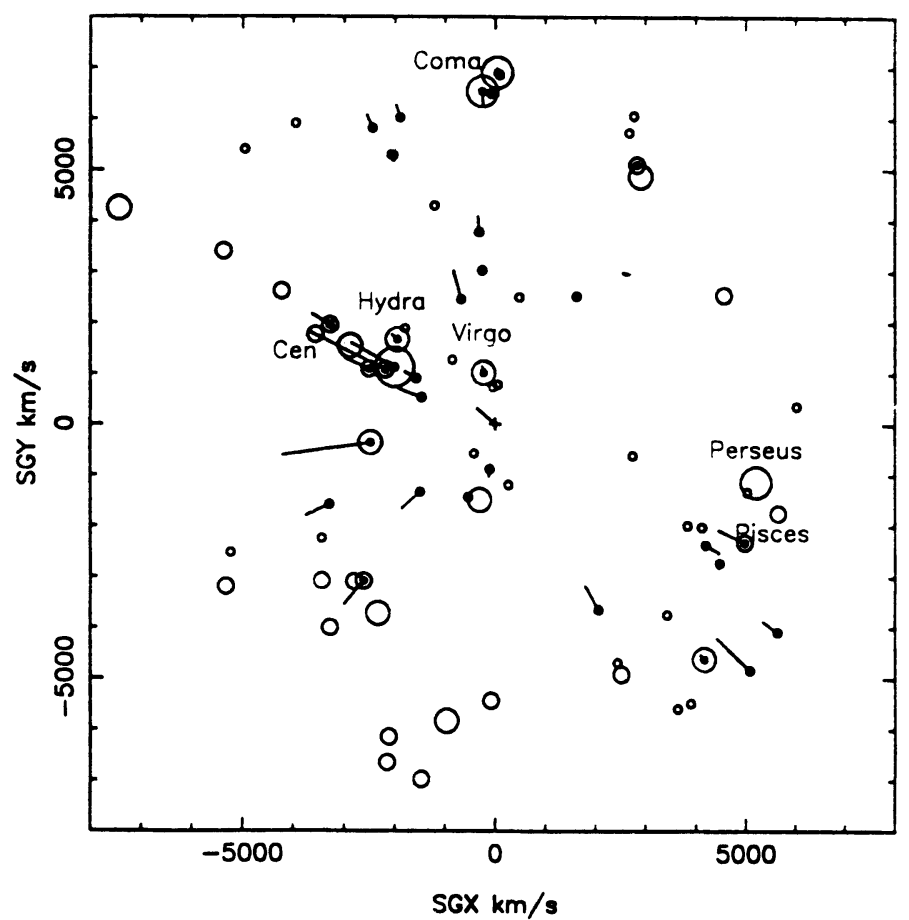

Figure 3. Peculiar velocities in the CMB frame for clusters and groups of galaxies with accurate Tully-Fisher distances. Major superclusters are labeled. The circle size indicates the richness of the cluster. The local group is at the origin, and the lines indicate the amplitude and direction of the peculiar motion. (From Mould et al. 1993)

sity regions because they are morphologically biased to large, dusty late type galaxies, but sample through the galactic plane moderately well (e.g. Figure 6).

The major IRAS surveys include the QDOT survey of Saunders et al. (1992) and Rowan-Robinson et al. (1990), the IRAS $1.936 \mathrm{Jy}$ sample of Strauss et al. $(1990 ; 1992 \mathrm{a} ; 1992 \mathrm{~b})$, the and the IRAS $1.2 \mathrm{Jy}$ sample of Fisher et al. (1995), which is soon to be published (e.g. Figure 6). The major optical galaxy surveys include the compilations of Lynden-Bell and Lahav (1989), Scharf and Lahav (1993) and Hudson (1993; 1994) primarily based on the optical radial velocity catalog of Huchra et al. (1992), and the new Optical Redshift Survey of Santiago et al. (1995), which defined a complete diameter limited sample in advance of obtaining redshifts for the galaxies in it. Both the optical galaxy surveys and IRAS surveys find essentially the same structures (viz Figs 5 and 6), although the density enhancements associated with any structure or cluster depends on the sample 


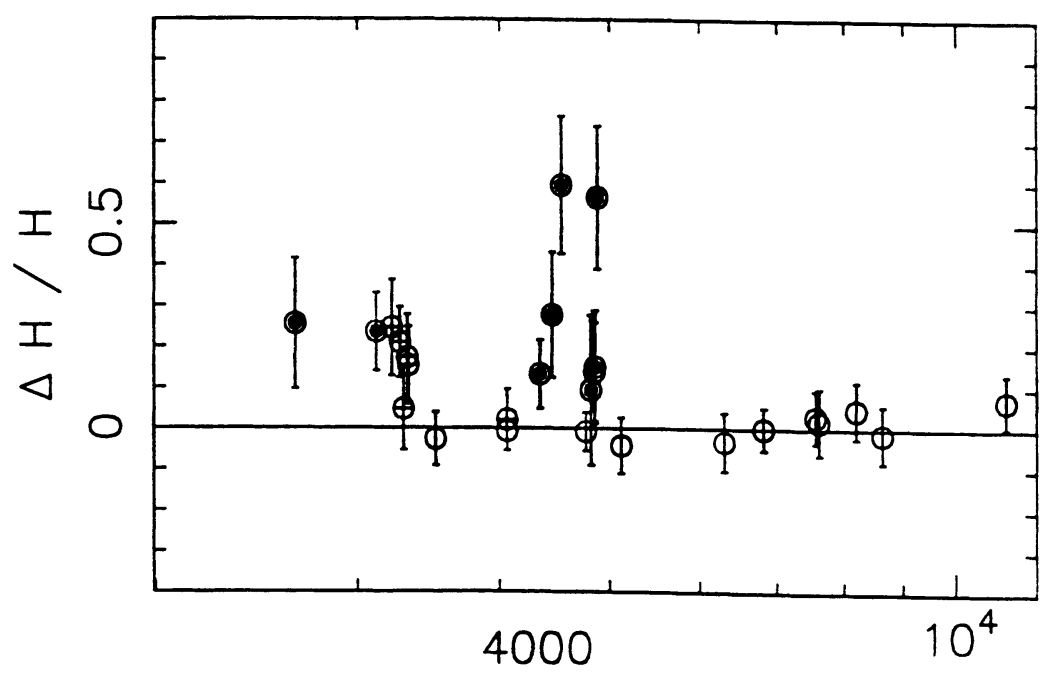

Radial velocity $(\mathrm{km} / \mathrm{s})$

Figure 4. Deviations from a uniform Hubble flow, $\Delta \mathrm{H} / \mathrm{H}$, in the CMB frame plotted against cluster redshift. The asymptotic $\mathrm{H}_{0}$ appears to be reached beyond $5,000 \mathrm{~km} / \mathrm{s}$. Note the "hiccup" in the flow near the velocity of Hydra-Centaurus. (From Mould et al. 1991).

used to define it. All major structures seen inside $10,000 \mathrm{~km} / \mathrm{s}$, probably even including the Great Wall (c.f. Mathewson and Ford 1994), appear to have some flows associated with them, albeit weak (Pisces-Perseus, Willick 1991). On very small scales (inside $3,000 \mathrm{~km} / \mathrm{s}$ ), Tully and Fisher's (1987) atlas has been an invaluable tool.

Major cluster surveys have been done by Postman, Huchra and Geller (1992) and Olowin et al. (1993), both to map the large-scale distribution of galaxy clusters and measure the amplitude of cluster clustering. Perhaps the most important aspect to the study of the cluster distribution has been the controversy started by Scaramella et al. (1989) and Tully and collaborators who have suggested that the bulk flow beyond the Great Attractor, and thus the missing few hundred $\mathrm{km} / \mathrm{s}$ of motion w.r.t. the CMB frame, is caused by the presence of the Shapley Supercluster on the line-of-sight to the GA but at a redshift of $\sim 14,000 \mathrm{~km} / \mathrm{s}$. By definition, this implies that the flow field doesn't converge till at least 15,000 km/s. Lauer and Postman's (1994) result is consistent with this lack of convergence nearby, but they do not find a significant motion w.r.t. the Shapley Supercluster, but it is near the edge of their sample. As usual, more work is needed! 


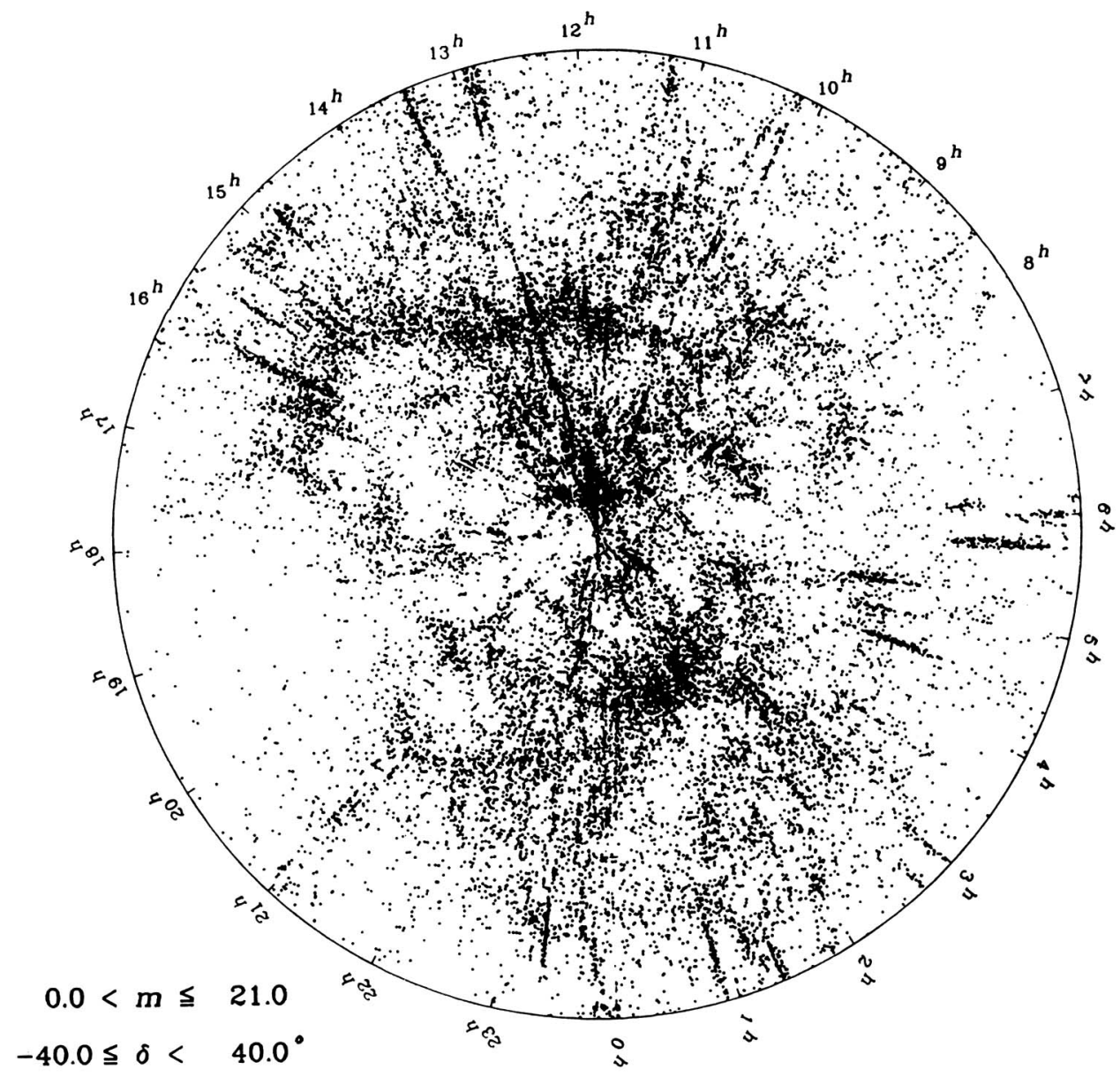

Figure 5. The latest galaxy map from the CfA Redshift Catalogue (Huchra et al. 1992) showing all galaxies with measured redshifts inside a wedge between declinations $-40^{\circ}$ and $+40^{\circ}$ and inside a radius of $15,000 \mathrm{~km} / \mathrm{s}$. The major well known structures such as the Great Wall between $8^{h}$ and $17^{h}$ at $8,000 \mathrm{~km} / \mathrm{s}$, Perseus-Pisces between $0^{h}$ and $5^{h}$ and at $4,000 \mathrm{~km} / \mathrm{sec}$, and Hydra-Cen, between $10^{h}$ and $14^{h}$ at $\sim 3,800 \mathrm{~km} / \mathrm{s}$, are easily seen. Since all galaxies with measured redshifts are plotted, well studied clusters of galaxies, which appear as "Fingers of God," are over represented.

Significantly deeper surveys are now underway. The QDOT2 survey of Saunders and collaborators will contain well over 10,000 galaxies over the whole sky while optical surveys based onthe extension of the Southern Sky Redshift Survey (the SSRS2) and the CfA redshift survey (daCosta et al. 1994; Geller and Huchra 1989) 


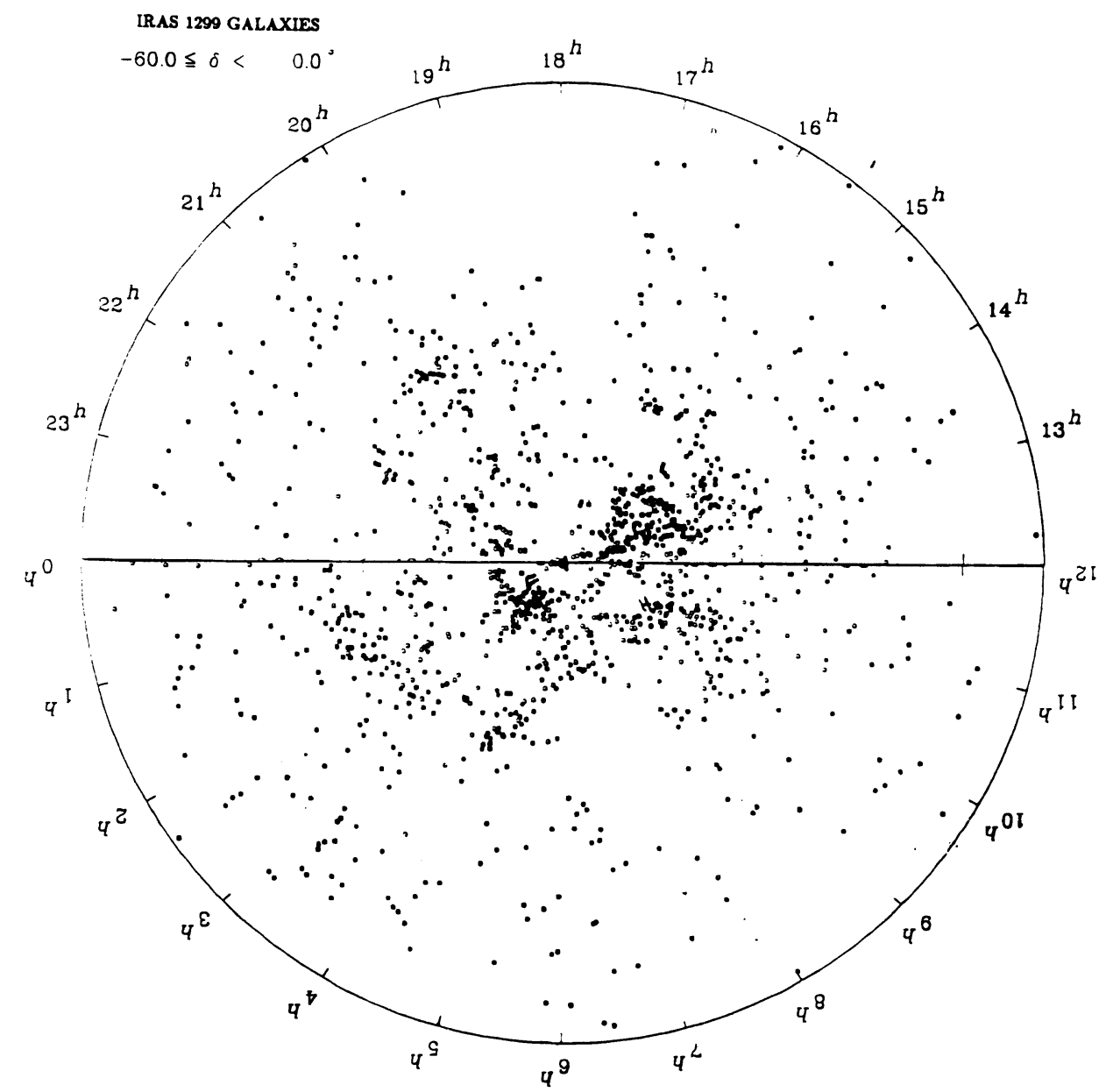

Figure 6. Galaxy map in the southern celestial hemisphere, declinations $-60^{\circ}$ to $0^{\circ}$ and within $12,000 \mathrm{~km} / \mathrm{s}$ from the IRAS $1.2 \mathrm{Jy}$ survey of Strauss et al. 1995). Hydra-Centaurus is easily seen as in Figure 5; the cluster on the other side of the sky $\left(2.5^{h}\right.$ and $\left.1,000 \mathrm{~km} / \mathrm{s}\right)$ is Fornax.

\section{CURRENT STATUS - What Exactly Do We Know?}

We can summarize the current status of our knowledge of nearby galaxy motions relatively quickly - although I think you'll be brought more up to date in the next few talks!

On the question of convergence, we don't yet know the answer. There are some indications that convergence has occurred by about $10,000 \mathrm{~km} / \mathrm{s}$ (to wit the most recent IRAS and POTENT matches), but the results of 
Lauer and Postman (1994) as well as the earlier conjectures of Scaramella et al. (1989) strongly indicate that we have not yet seen convergence on scales of nearly $15,000 \mathrm{~km} / \mathrm{s}$. This question also remains bound up with the determination of the maximum scales (both dimension and mass) on which large scale clustering occurs (c.f. Park et al. et al. 1994).

Can we explain most of the CMB motion locally? Maybe! Mould et al. (1993) find several possible models that fit the data: a Great Attractor model, a Bulk Flow Model, a Bi-infall Model, and a Moving Attractor model all give acceptable fits to the available data with velocities and directions "consistent" with the CMB motion.

What are such galaxy motions effects on the determination of $\mathrm{H}_{0}$ ? They are still too large since almost all of the calibrating galaxies for SN, IRTF, etc. are in the flow field (e.g. in the Virgo Cluster core), but this situation is rapidly being improved by HST Cepheid distances (e.g. Freedman et al. 1994). There are still significant discrepancies between $\mathrm{H}_{0}$ derived from different distance indicators, but as better and better cross-calibrations are performed, these differences are likely to go away. Indeed, the most recent such cross-calibration based on the Cephied distance to Virgo (Mould et al. 1995) indicates that the best local value of $\mathrm{H}_{0}$ is near $80 \mathrm{~km} / \mathrm{s} / \mathrm{Mpc}$ independent of the distance measuring technique used to step beyond Virgo.

The density field is somewhat better known, at least out to moderate distances and above the plane. The surveys mentioned above, ORS, CfA2, SSRS2, and QDOT2, are providing reasonable maps to $8,000 \mathrm{~km} / \mathrm{sec}$. The IRAS-POTENT match (Dekel et al. 1993) is pretty good but very limited because the IRAS catalogs pnly sparsely sample the density field so that flows on linear scales smaller than $1000-1200 \mathrm{~km} / \mathrm{sec}$ are lost in the smoothing of the data - Virgo flow is only marginally detectable.

Perhaps the biggest problem comes from the discrepancy between the IRAS and optical survey matches of the velocity and density field. From the $1.936 \mathrm{Jy}$ survey data, Dekel et al. (1993) found $\Omega \mathrm{b}(\mathrm{IRAS})=1.3_{-0.6}^{+0.7}$ (where $\Omega$ is, of course, the ratio of the mass density to the cosmological closure mass density, and $\mathrm{b}$ is the biasing factor of Bardeen et al. 1986). Preliminary results from the 1.2Jy sample indicate that this number might be somewhat smaller $-\Omega \mathrm{b}(\mathrm{IRAS}) \sim 0.8 \pm 0.4$. However the optically selected galaxy density field gives $\Omega \mathrm{b}$ (Optical) $\sim 0.3 \pm 0.1$ (Hudson 1993; 1994), similar to the values derived earlier for Virgo infall (Huchra 1988). This is a problem that absolutely must be understood in order to settle the $\Omega$ debate as well as to understand if "biased" galaxy formation is required to fit the observations.

Lastly, it is interesting to point out that new, and possibly massive, nearby objects are being found every year (Kraan-Korteweg et al. 1994). Our census of the nearby universe, both in terms of the density field and the 
velocity field still needs a lot of work. A number of projects are underway to improve this situation, for example the 2 Micron All Sky Survey and DENIS, which will produce significantly better galaxy catalogues for density field studies as well as calibrated IR magnitudes for use in some of the distance estimating programs, and the HST Cephied program which should provide a good cross-calibration of many distance measuring techniques thus producing better maps of the flow field. The "Warpfire" project of Lauer and Postman is proceeding with theuse of clusters to map the flow field, albeit sparsely, to greater and greater distances. We need to complete these programs and get on with the systematic measurement of galaxy distances to $\mathrm{z} \sim 0.05$ and beyond.

As has been concluded in the recent review of Dekel (1994), there are large-scale motions in the Universe. We are just beginning to map them in nearby space, but are far from having a complete understanding even nearby. Wish us luck!

This work has been supported in part by NASA grant GO-2227 from StScI and by the Smithsonian Institution. The author would like to thank his collaborators in the Hubble Constant/Large-Scale Flow project, especially J. Mould, W. Freedman, G. Bothun, R. Schommer, M. Postman, T. Lauer, J. Tonry, R. Marzke, M. Geller M. Strauss, A. Yahil, O. Lahav, and M. Davis, as well as fellow travellers A. Dressler, D. Burstein, G. Wegner, D. Lynden-Bell, G. de Vaucouleurs, S. Van den Bergh, D. Mathewson, S. Courteau, J. Willick, A. Sandage and G. Tammann, without whose interactions this work would never have been done. Special thanks go to Marc Aaronson, a friend whose drive and vision, cut short all too early, started this all.

\section{References}

Aaronson, M., Bothun, G. Mould, J., Huchra, J. Schommer, R. and Cornell, M. 1986, ApJ302,536.

Aaronson, M., Huchra, J., Mould, J., Schechter, P. and Tully, R. B. 1982,ApJ,258,64.

Aaronson, M., Mould, J., Huchra, J. Sullivan, W., Schommer, R. and Bothun, G. $1980, \mathrm{ApJ}, 239,12$.

Bardeen, J., Bond, J. R., Kaiser, N. and Szalay, A, 1986, ApJ304,15

Bertschinger, E. and Dekel, A. 1989, ApJL,336,L5.

Burstein, D. Davies, R., Dressler, A., Faber, S., Lynden-Bell, D., Terlevich, R. and Wegner, G. 1986, in Galaxy Distances and Deviations from Universal Expansion, Madore and Tully, eds. (Dordrecht: Reidel).

Cheng, E., Saulson, P., Wilkinson, D. and Corey, B. 1979,ApJ,232,L139.

Courteau, S. 1992, PhD Thesis, UC Santa Cruz.

Courteau, S, Faber, S., Dressler, A. and Willick, J. 1993, ApJL,412,L51.

daCosta, L., Vogeley, M., Geller, M., Huchra, J. and Park, C. 1994, ApJL, in press.

Davis, M., Huchra, J., Latham, D. and Tonry, J. 1982,ApJ,253,423.

Dekel, A. 1994, Ann. Rev. Astron. and Ap. 32,371.

Dekel, A., Bertschinger, E. and Faber, S. 1990, ApJ364,349. 
de Vaucouleurs, G. 1958,AJ,63,253.

Dressler, A. and Faber, S. 1991, ApJ,368,54.

Fisher, K. et al. 1995, ApJ, in prep.

Freedman, W., Madore, B., Mould, J., Hill, R., Ferrarese, L., Kennicutt, R., Saha, A., Stetson, P., Graham, J., Ford, H., Hoessel, J., Huchra, J., Hughes, S and Illingworth, G. 1994, Nature,371,757.

Geller, M. and Huchra, J. 1989,Science,246,897.

Hamuy, M., Phillips, M., Maza, J., Suntzeff, N., Schommer, R. and Avilés, R. 1995, AJ, in press.

Huchra, J. 1988, in The Extragalactic Distance Scale, van den Bergh and Pritchet, eds., p257.

Huchra, J., Geller, M., Clemens, C., Tokarz, S., and Michel, A. 1992, Bull.CDS,41,31.

Hudson, M. 1993, MNRAS, 265,72.

Hudson, M. 1994, MNRAS,266,475.

Kirshner, R., Oemler, A., Schechter, P. and Shectman, S. 1981,ApJL,248,L57.

Kraan-Korteweg, R., Loan, A., Burton, W., Lahav, O., Ferguson, H., Henning, P. and Lynden-Bell, D. 1994, Nature,372,77.

Lauer, T. and Postman, M. 1994, ApJ,425,418.

Lynden-Bell, D., Faber, S., Burstein, D. Davies, R., Dressler, A., Terlevich, R. and Wegner, G. 1988,ApJ,326,19.

Lynden-Bell, D. and Lahav, O. 1989, in Large Scale Motions in the Universe, Rubin and Coyne, eds, (Princeton: Princeton), p199.

Mathewson, D., Ford, V. and Buchhorn, M. 1992, ApJL,389,L5.

Mathewson, D. and Ford, V. 1994, ApJL,434,L39.

Mould, J., Akeson, R., Bothun, G., Han, M., Huchra, J., Roth, J. and Schommer, R. 1993, ApJ409,14.

Mould, J., Stavely-Smith, L., Schommer, R., Bothun, G., Hall, P., Han, M., Huchra, J., Roth, J., Walsh, W. and Wright, A. 1991, ApJ,383,467.

Mould, J. et al., 1995, ApJ, in prep.

Olowin, R., Huchra, J. and Corwin, H. 1993, in Observational Cosmology, Chincarini et al., eds, p104.

Park, C., Vogeley, M., Geller, M. and Huchra, J. 1994,ApJ,431,596.

Peebles, P. J. E. 1976,ApJ,205,318.

Postman, M., Huchra, J. and geller, M. 1992, ApJ,384,404.

Reiss, A., Press, W. and Kirshner, R. 1995,ApJ, in press.

Rowan-Robinson, M., Lawrence, A., Saunders, W., Crawford, J., Ellis, R. Frenk, C., Parry, I., Xiaoyang, X., Allington-Smith, J., Efstathiou, G., and Kaiser, N. 1990, MNRAS, 247,1 .

Rubin, V. 1951, MSc thesis, Cornell University.

Rubin, V., Ford, W., Thonnard, N., Roberts, M. and Graham, J. 1976a, AJ,81,687.

Rubin, V., Thonnard, N., Ford, W. and Roberts, M. 1976b, AJ,81,719.

Sandage, A. and Hardy, E. 1972,ApJ,172,253.

Sandage, A. and Tammann, G. 1981, A Revised Shapley Ames Catalog of Bright Galaxies (Washington: Carnegie Institution).

Santiago, B, Strauss, M., Lahav, O., Davis, M., Dressler, A. and Huchra, J. 1995, ApJ, in press.

Saunders, W., Rowan-Robinson, M., and Lawrence, A. 1992, MNRAS,258,134.

Scaramella, R., Baiesi-Pillastrini, G., Chincarini, G. Vettolani, G. and Zamorani, G. 1989, Nature, 338,562 .

Scharf, C. and Lahav, O. 1983, MNRAS,264,439.

Schmidt, B., Kirshnerm R., Eastman, R., Phillips, M., Suntzeff, N., Hamuy, M., Maza, J. and Avilés, R. 1994, ApJ,432,42.

Shaya, E. 1984,ApJ,280,470.

Silk, J. 1974,ApJ,193,525.

Smoot, G. and Lubin, P. 1979,ApJ234,L83. 
Strauss, M., Davis, M., Yahil, A. and Huchra, J. 1990, ApJ,361,49.

Strauss, M., Davis, M., Yahil, A. and Huchra, J. 1992a, ApJ,385,421.

Strauss, M., Huchra, J., Davis, M., Yahil, A., Fisher, K. and Tonry, J. 1992b, ApJS,83,29.

Tammann, G. and Sandage, A. 1985,ApJ,294,81.

Tonry, J. and Davis, M. 1980,ApJ,246,680.

Tonry, J. and Schneider, D. 1988, AJ,96,807.

Tully, R. B. and Fisher, R. 1987, Nearby Galaxy Atlas, Cambridge: Cambridge).

Tully, R. B and Shaya, E. 1984,ApJ281,31.

Willick, J. 1991, PhD Thesis, UC Berkeley. 